Relations industrielles

Industrial Relations

\title{
Hébert, Gérard, Traité de négociation collective
}

\section{Reynald Bourque}

Volume 48, numéro 2, 1993

URI : https://id.erudit.org/iderudit/050859ar

DOI : https://doi.org/10.7202/050859ar

Aller au sommaire du numéro

Éditeur(s)

Département des relations industrielles de l'Université Laval

ISSN

0034-379X (imprimé)

1703-8138 (numérique)

Découvrir la revue

Citer ce compte rendu

Bourque, R. (1993). Compte rendu de [Hébert, Gérard, Traité de négociation collective]. Relations industrielles / Industrial Relations, 48(2), 355-358.

https://doi.org/10.7202/050859ar

Tous droits réservés @ C Département des relations industrielles de l'Université Laval, 1993
Ce document est protégé par la loi sur le droit d'auteur. L'utilisation des services d'Érudit (y compris la reproduction) est assujettie à sa politique d'utilisation que vous pouvez consulter en ligne.

https://apropos.erudit.org/fr/usagers/politique-dutilisation/ 


\section{RECENSIONS BOOK REVIEWS}

Traité de négociation collective, par Gérard HéBERT, Boucherville, Gaétan Morin Éditeur, 1992, 1242 p., ISBN 2-89105-433-4

Le Traité de négociation collective que vient de publier le professeur Gérard Hébert est un ouvrage monumental, à l'image de la carrière universitaire de son auteur qui a enseigné pendant plus de vingt-cinq ans à l'École de relations industrielles de l'Université de Montréal. Cet ouvrage comptant 1242 pages et 33 chapitres s'avère d'ores et déjà indispensable à tout enseignement universitaire sur la négociation et les conventions collectives au Québec. Comme son titre l'indique, l'ouvrage du professeur Hébert se veut un «traité » c'est à dire « un ouvrage didactique où est exposé d'une manière systématique un sujet ou un ensemble de sujets concernant une matière » (Petit Robert), ou encore pour reprendre les termes de l'auteur « la somme des connaissances sur un sujet donné » (Avant-propos). Il aurait été plus juste de l'intituler « Traité de la négociation collective dans les contextes québécois et nord-américain », puisqu'il sagit d'un ouvrage voué essentiellement à l'analyse descriptive des dispositifs conventionnels et des différents régimes de négociation collective au Québec, étayé de nombreuses références aux situations canadienne et américaine à ce chapitre.

L'ouvrage débute par deux chapitres préliminaires, l'un présentant la nature et les objectifs généraux de la négociation collective, l'autre situant les contextes historique, légal et socio-économique de la négociation collective au Canada et au Québec. Le reste de l'ouvrage couvrant différentes facettes du phénomène de la négociation collective est divisé en trois parties: l'objet et le contenu des conventions collective; le processus de négociation; les aspects généraux et les problèmes actuels de la négociation collective.

La première partie de l'ouvrage, consacrée à l'objet de la négociation collective, brosse en quatorze chapitres un tableau très exhaustif des principales conditions de travail contenues dans les conventions collectives au Québec. Le chapitre 3 présente le rôle, le statut juridique et la répartition des conventions collectives de 1984 à 1989 au Québec par secteurs d'activités, régions et centrales syndicales. L'auteur a eu l'heureuse idée d'annexer à ce chapitre des notes techniques sur la codification, les banques de données et les autres sources d'information relatives aux conventions collectives au Canada et au Québec. Chacun des douze chapitres suivants scrute un domaine spécifique de la couverture conventionnelle, passant en revue le cadre légal, les dispositions les plus courantes, les problèmes et les enjeux particuliers caractérisant les négociations collectives en cette matière. Les sujets de négociation analysés dans ces chapitres sont la sécurité syndicale, les droits de gérance, le règlement et l'arbitrage des griefs, les mesures disciplinaires, l'ancienneté et les mouvements de personnel, la protection de l'emploi, le contenu et l'évaluation des tâches, les heures de travail, les salaires, les avantages sociaux, la durée et le renouvellement des conventions collectives. Un dernier chapitre 
explorant les tendances et les orientations de la convention collective vient clore cette première partie.

La deuxième partie de l'ouvrage analyse en douze chapitres le processus de la négociation collective, l'emphase étant mise sur les structures et les différents régimes caractérisant le Québec. Le chapitre 17, qui sert d'introduction à cette partie, présente brièvement les éléments constitutifs du processus de la négociation collective et les particularités à cet égard du secteur public et parapublic, de même que les règles s'appliquant aux cadres et professionnels conventionnés de ce secteur. Les trois chapitres suivants s'intéressent au processus de structuration interne et externe de la négociation collective. D'abord, l'auteur approfondit la distinction classique entre unité légale, unité réelle et unité d'influence en soulignant l'importance des rapports de pouvoir dans la détermination des unités de négociation. Il décrit ensuite les principaux types d'unités d'influence en fonction du degré d'uniformisation et de contrainte qu'ils comportent : contrats uniformes couvrant un secteur ou une profession, contrat cadre, négociations coordonnées et négociation type (pattern bargaining). Il s'agit là, note l'auteur, de structures types susceptibles de combinaisons et de variations multiples dans la réalité. Le dernier chapitre de cette trilogie retrace les grandes lignes du débat sur la négociation sectorielle au Québec depuis les années 1960, soupesant les implications et les problèmes d'une formule qui n'emporte manifestement pas l'adhésion de l'auteur.

Les chapitres 21 à 24 examinent le processus et les méthodes de la négociation collective directe, le rôle des tiers-intervenants dans le règlement des conflits d'intérêt, les causes et les effets des conflits du travail. Cet ensemble débute avec un chapitre décrivant les principales étapes, les stratégies et les tactiques de la négociation collective, inspiré largement des travaux classiques sur le sujet, notamment ceux de Chamberlain et Stevens. Vient ensuite un excellent chapitre traitant des coûts de la convention collective préparé par Patrice Jalette, étudiant au doctorat à l'École de relations industrielles de l'Université de Montréal. Cette contribution a le mérite de clarifier les objectifs et d'offrir de nombreux exemples des techniques de l'évaluation des coûts de la convention collective, comblant ainsi une lacune manifeste dans les ouvrages en langue française sur la négociation collective. Le chapitre consacré au règlement des conflits situe le cadre légal de la conciliation, de la médiation et de l'arbitrage au Québec en comparaison avec les autres compétences canadiennes et fait une large part à la discussion des modes de règlement des conflits d'intérêt public. Au chapitre 24, l'auteur analyse les dimensions juridiques, économiques et sociologiques des conflits du travail et procède à une comparaison des statistiques québécoises, canadiennes et internationales en cette matière, confirmant le déclin généralisé du recours à la grève, au cours des années 1980, dans les principaux pays industrialisés.

Poursuivant la seconde partie de l'ouvrage, les chapitres 25 à 28 forment une série d'études bien documentées sur les régimes de négociation collective dans les secteurs public et parapublic et dans d'autres secteurs assujettis à une réglementation en marge du régime commun des rapports collectifs du travail au Québec. Le chapitre 25 discute les problèmes généraux de la négociation collective et du règlement des différends dans les services publics et examine de ce point de vue les régimes caractérisant la fonction publique fédérale, le secteur municipal canadien et les principales instances du secteur public aux États-Unis. Le chapitre suivant rappelle l'évolution législative et le cadre 
actuel des relations du travail dans les secteurs public et parapublic au Québec, décrivant avec minutie les unités de négociation, la représentation syndicale et les modes de fonctionnement spécifiques dans chacun des sous-secteurs. En complément à cette étude fouillée, un autre chapitre s'attarde à la situation des cadres et des professionnels conventionnés du secteur public au Québec regroupés en de nombreuses associations intégrées par voie consultative au processus de réglementation des conditions de travail régissant ces différentes catégories de personnel. Cette seconde partie s'achève sur un chapitre dédié aux régimes des décrets, de l'industrie de la construction, des professionnels et cadres en dehors du secteur public.

La troisième partie comporte cinq chapitres abordant les aspects généraux théoriques et analytiques - de la négociation collective. Le chapitre 29 passe en revue les théories et les modèles qui ont marqué l'étude du phénomène de la négociation collective et propose une typologie distinguant trois approches - économique, mixte et institutionnelle - parmi les principales contributions théoriques dans ce domaine. Le chapitre qui suit esquisse à grands traits les régimes de négociation collective en GrandeBretagne, en France et en Suède, cherchant ensuite à dégager, à travers une comparaison avec l'Allemagne, la Belgique et l'ltalie, les similitudes et les différences significatives à cet égard dans les pays européens. Les trois derniers chapitres de l'ouvrage s'apparentent à une conclusion générale. Au chapitre 31, l'auteur analyse et discute les problèmes actuels de la négociation collective dans un contexte de changements technologiques accélérés, de récession, de libre-échange et de déréglementation. Il enchaîne avec un chapitre soulignant les principales initiatives gouvernementales et les différentes formules mises en œuvre pour promouvoir la concertation patronale-syndicale aux ÉtatsUnis, au Canada et au Québec au cours des trois dernières décennies. Le chapitre final extrapole les tendances prévisibles de la négociation collective dans les secteurs privé et public au Canada et au Québec, l'auteur entrevoyant un avenir prometteur dans le premier cas et des difficultés dans le secteur public en raison des atavismes qui mineraient selon lui les relations entre les parties.

Que dire d'un ouvrage de cette envergure, dont la lecture intégrale constitue en soi un exploit? Il est difficile de porter un jugement global, compte tenu de la diversité et de la complexité des sujets traités par l'auteur. Soulignons d'abord la facture impressionnante de l'ouvrage, tant par son ampleur et son contenu que par sa qualité rédactionnelle. De lecture agréable, clair, précis dans les moindres détails, il est également bien servi par une table des matières élaborée et une bibliographie sélective à la fin de chacun des chapitres. On ne peut que s'incliner devant l'énormité de la tâche accomplie par le professeur Hébert qui a bénéficié toutefois de l'appui de quelques collaborateurs. Ainsi, les premières versions du chapitre 8 sur les mesures disciplinaires, des chapitres 22 et 29 traitant des coûts de la convention et des théories de la négociation collective, et du chapitre 30 sur la négociation collective en Europe sont dues à Daniel Rochefort, Patrice Jalette et Carol Bélanger, respectivement avocat spécialisé en droit du travail chez Heenan, Blaikie et Associés, et étudiants au doctorat à l'École de relations industrielles de l'Univiersité de Montréal.

Bien que remarquable, l'ouvrage du professeur Hébert n'est pas parfait. Certains déploreront sans doute l'absence d'un cadre d'analyse explicite permettant d'intégrer les données empiriques et les descriptions détaillées des institutions dont l'ouvrage 
foisonne. Contrairement à d'autres auteurs, tels Kochan et Katz aux États-Unis et Craig au Canada, qui structurent leurs ouvrages en référence à un modèle ou un cadre d'analyse, le professeur Hébert a plutôt opté pour une démarche chère aux institutionnalistes depuis Commons, privilégiant l'analyse historique et descriptive. D'autres s'étonneront peut-être des prises de positions introduites ici et là par l'auteur afin, souligne-t-il dans son avant-propos, de stimuler la discussion. Les lecteurs assidus du professeur Hébert ne seront cependant pas surpris de retrouver dans cet ouvrage des idées qu'il véhicule depuis plus de vingt ans, telles sa conviction profonde des vertus du système nordaméricain de négociation collective en regard des systèmes plus centralisés des pays européens et scandinaves, et ses critiques acerbes du recours à la grève et de la centralisation bureaucratique des négociations collectives dans le secteur public.

On peut enfin se demander si certains chapitres ne sont pas carrément superflus dans un ouvrage de cette longueur. Les deux premières parties offrent à notre avis une synthèse originale de la portée, des problèmes et des enjeux majeurs de la négociation collective dans les secteurs privé et public au Québec, mais l'utilité de la troisième partie nous semble moins évidente. Il existe de nombreux ouvrages et articles sur les comparaisons internationales et les théories de la négociation collective, et les trois derniers chapitres de l'ouvrage auraient pu tenir lieu de conclusion générale.

Malgré ces quelques réserves, l'ouvrage du professeur Hébert nous apparait comme une contribution magistrale et incontournable, destinée à accompagner tout au long de leur cursus universitaire les étudiantes et étudiants inscrits à des programmes spécialisés en relations industrielles. Les enseignants et enseignantes y trouveront également leur compte, puisque la publication de l'ouvrage de Gérard Hébert vient régler l'éternel problème du choix d'un manuel de référence pour l'enseignement de la négociation collective dans les universités québécoises.

Reynald Boukque

Université de Montréal et Université du Québec à Hull

The Future of Industrial Relations: Proceedings of the Second Bargaining Group Conference, by Harry C. KATZ, ed., Institute of Collective Bargaining, New York State School of Industrial and Labor Relations, Cornell University, 1991, 169 p., ISBN 0-87546-850-0.

Harry Katz introduces this edited volume by observing that it emerged from the Second Bargaining Conference held in the summer of 1990 at the New York State School of Industrial and Labor Relations (Cornell University). This conference serves as a major impetus for our continued effort to understand trends in industrial relations. This book represents a growing trend to publish conference proceedings as books and contains fifteen original papers written by expert contributors.

The book is very impressive, and most of my review will reflect this attitude. I will first describe the book's objectives and its intended audience, then briefly describe and critique each section, and finally, conclude with some general observations. 